\title{
The Sivers and other semi-inclusive single-spin asymmetries at HERMES
}

\author{
Gunar Schnell [For the HeRmes Collaboration] \\ DESY, 15738 Zeuthen, Germany \\ E-mail: gunar.schnell@desy.de
}

\begin{abstract}
Azimuthal single-spin asymmetries of lepto-produced pions and charged kaons were measured on a transversely polarized hydrogen target. Evidence for a naive-T-odd, transverse-momentumdependent parton distribution function is deduced from non-vanishing Sivers effects for $\pi^{+}, \pi^{0}$, and $K^{ \pm}$, as well as in the difference of the $\pi^{+}$and $\pi^{-}$cross sections. Further azimuthal modulations of the single-spin asymmetry were found to be consistent with zero except the one related to the Collins effect and one that is sub-leading in an expansion of the cross section in $1 / Q$.
\end{abstract}

XVIII International Workshop on Deep-Inelastic Scattering and Related Subjects April 19 -23, 2010

Convitto della Calza, Firenze, Italy 


\section{Sivers asymmetry}

Ten years have passed since the Hermes Collaboration measured target-spin-dependent azimuthal distributions of pions produced in deepinelastic scattering (DIS) [1]. While first interpretations of those data focussed on the Collins effect [2]-a left-right asymmetry, with respect to the transverse spin of a fragmenting quark, in the momentum distribution of the produced hadrons - the seminal paper [3] demonstrated that also an asymmetric momentum distribution of unpolarized quarks in transversely polarized nucleons can lead to such azimuthal distributions not suppressed by $1 / Q$. When scattering off longitudinally polarized nucleons, this Sivers effect is indistinguishable from the Collins effect and only data using transversely polarized nucleons could give the final verdict on the origin of the azimuthal dependences observed.

The Sivers parton distribution function (PDF) is just one example of several PDFs that parametrize correlations between the parton's transverse momentum and the parton's and/or nucleon's spin. Among those the Sivers function relates to the distribution of unpolarized quarks in a transversely polarized nucleons and is rather particular as it is naive-T-odd,
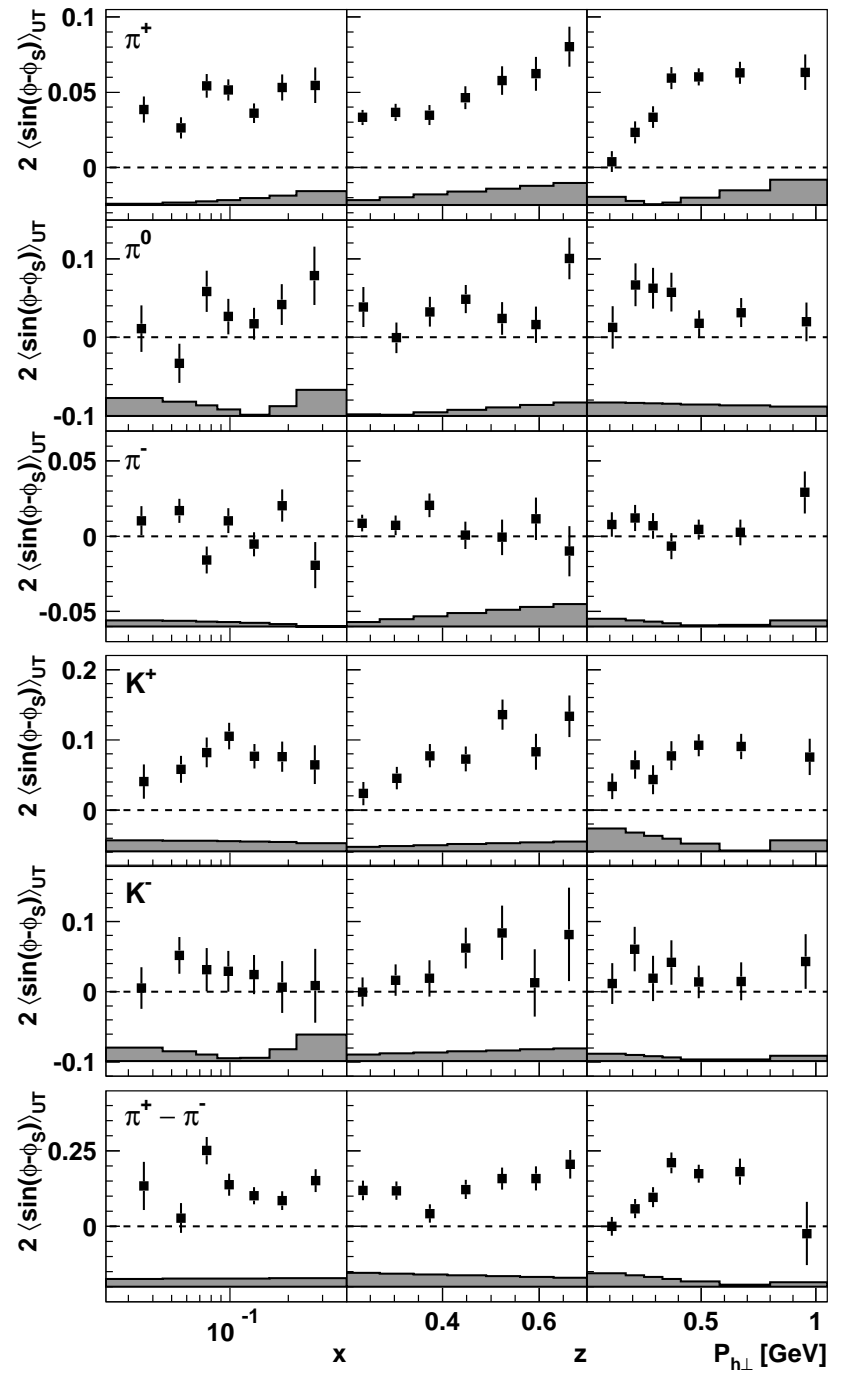

Figure 1: Sivers amplitudes for pions, charged kaons, and the pion-difference asymmetry (as denoted in the panels) as functions of $x, z$, or $P_{h \perp}$. The systematic uncertainty is given as a band at the bottom of each panel. In addition there is a $7.3 \%$ scale uncertainty from the target-polarization measurement.

thus requiring in DIS final-state interactions. It breaks the conventional understanding and interpretation of factorization and universality. If measured in Drell-Yan, QCD quite firmly predicts the Sivers function to be of opposite sign as in DIS. In $p p \rightarrow h X$ no firm prediction can even be made at all at present.

Nevertheless, the situation is rather straight-forward in an DIS experiment: one needs to measure the azimuthal distribution of hadrons produced in the scattering of unpolarized leptons by transversely polarized nucleons. When polarized transverse to the virtual-photon momentum di- 
rection five distinct Fourier modulations of the cross section can be identified (cf., e.g., Ref. [4]). The $\sin \left(\phi-\phi_{S}\right)$ modulation $^{1}$ is the signature of the Sivers effect while, e.g., the $\sin \left(\phi+\phi_{S}\right)$ modulation arises through the interplay of transversity and the Collins fragmentation function. Other modulations involve the pretzelosity distribution and genuine twist- 3 contributions. When the target is polarized perpendicular to the beam direction a sixth modulation arises from the small but non-vanishing longitudinal component of the target spin w.r.t. the momentum direction of the virtual photon. This $\sin \left(2 \phi+\phi_{S}\right)$ modulation is sensitive to one of the worm-gear distributions.

The HERMES experiment [6] took data with transversely polarized protons and the $27.5 \mathrm{GeV}$ $e^{+} / e^{-}$beam at HERA during the years 2002-2005. The excellent particle identification allowed for measurements of the azimuthal modulations in the cross section for pions as well as for charged kaons. In Fig. 1 the Sivers, i.e., the $\sin \left(\phi-\phi_{S}\right)$, amplitudes are presented for pions, charged kaons, and for the charged-pion cross-section difference [7]. Clear evidence for a non-vanishing Sivers function can be deduced from the significantly positive amplitudes for all but the $\pi^{-}$. These results lead to Sivers distributions that are opposite in sign for $\mathrm{u}$ - and d-quarks.

A puzzling facet of the data is the difference in magnitude of the amplitudes for $\pi^{+}$and $\mathrm{K}^{+}$. On the basis of u-quark dominance, e.g., the dominant contribution of u-quark scattering to the production of $\pi^{+}$and $K^{+}$, one would naively expect amplitudes of similar size, while in reality the $K^{+}$amplitudes are partially double in size of the $\pi^{+}$amplitudes. One apparent difference between the two mesons are their different valence structures: besides the u-quark, which is a valence quark in the target nucleon as well, the $\pi^{+}$is made of an anti-d quark in contrast to the anti-s quark for the $K^{+}$, both being sea quarks in the target nucleon. The question to ask therefore is whether there can be a significantly different role of the various sea quarks in the Sivers effect? One hint might come from an earlier result [8] by HERMES, the distribution of strange quarks in nucleons. It was found to be much softer than the one for the light sea, with the difference being largest where also the difference between the $\pi^{+}$and $K^{+}$Sivers amplitudes is the biggest (c.f. Figs. 2 (left) and 3). ${ }^{2}$

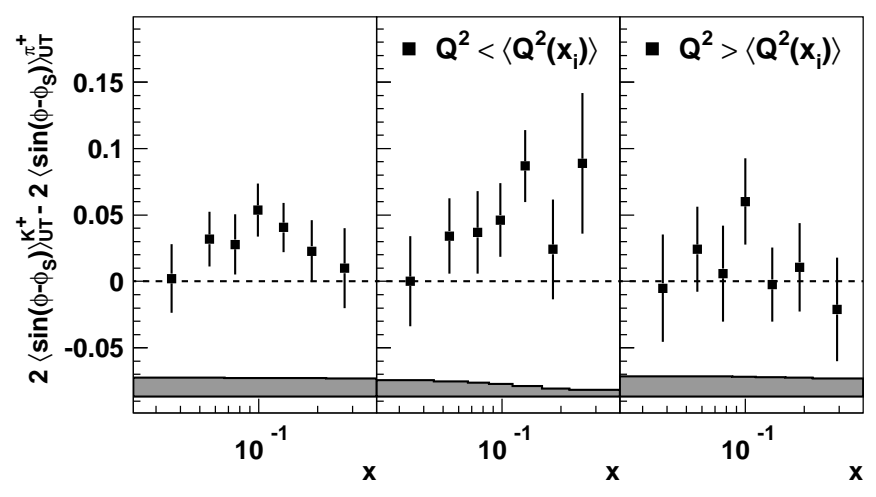

Figure 2: Difference of Sivers amplitudes for $K^{+}$and $\pi^{+}$as functions of $x$ for all $Q^{2}$ (left), and separated

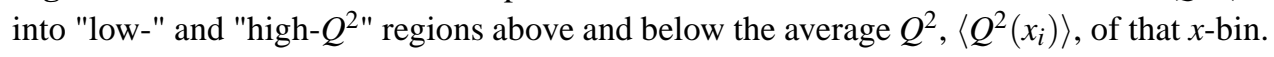

\footnotetext{
${ }^{1}$ All angles and asymmetries are defined in line with the Trento Conventions [5]. In particular, $\phi\left(\phi_{S}\right)$ is the azimuthal angle of the hadron momentum (the target-spin vector) about the virtual photon direction w.r.t. the lepton scattering plane.

${ }^{2}$ It is interesting to note that it is sufficient to have Sivers functions for sea quarks that are opposite in sign of the one for u-quarks to explain the $\pi^{+} / K^{+}$difference: the respective sea-quark contribution to $K^{+}$production will reduce the contribution from u-quarks less than to $\pi^{+}$production as there are fewer anti-s than anti-d quarks in the proton.
} 


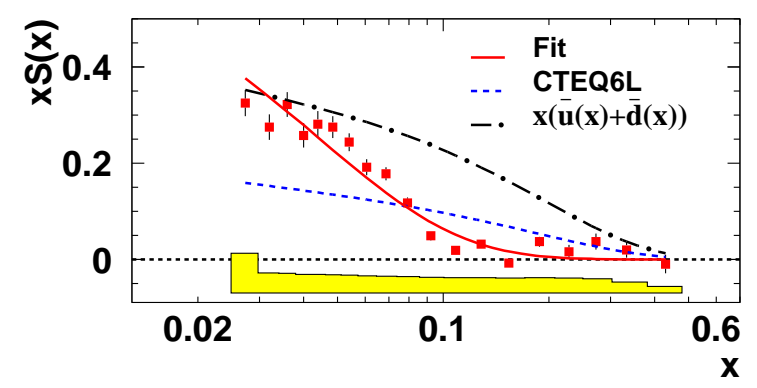

Figure 3: The strange-quark distribution $S(x) \equiv s(x)+\bar{s}(x)$ as a function of $x$ and compared to its CTEQ6L parameterization as well as to the light sea.
There are other aspects that need to be taken into account in the $\pi^{+} / K^{+}$ comparison. Even in the case of scattering solely off $\mathrm{u}$-quarks the role of the fragmentation function cannot be neglected as the fragmentation function appears in different convolutions over intrinsic and fragmentation quark transverse momenta in the numerator and denominator of the asymmetry. For example, varying dependences of the fragmentation functions on transverse momentum can lead to varying magnitudes of the asymmetry amplitudes. Another crucial aspect may also lead to the differences observed: unrelated $1 / Q^{2}$-suppressed contributions to the amplitudes. Indeed, looking at the $Q^{2}$ dependence of the $K^{+}-\pi^{+}$difference, the latter seems to be significantly non-zero at lower values of $Q^{2}$ only (Fig. 2). In addition, while there is no evidence for any $Q^{2}$ dependence of the $\pi^{+}$amplitudes there is a hint of systematically smaller $K^{+}$Sivers amplitudes at larger values of $Q^{2}$ (Fig. 4).

An entirely different azimuthal modulation is the $\sin \phi_{S}$ modulation. It receives subleadingtwist contributions only, but nevertheless was found to be non-zero-though decreasing with $Q^{2}$ for the $\pi^{-}$(Fig. 4 right). It can be related to several interesting distribution and fragmentation functions, e.g., transversity in conjunction with the novel interaction-dependent fragmentation function $\tilde{H}$, but also to the Sivers function or to the worm-gear distribution correlating the longitudinal quark and transverse nucleon polarizations. While disentangling these contribution will require further detailed studies, a rather interesting aspect can already be highlighted. The inclusive analogue, i.e., summing over all final-state hadrons and integrating over their four-momenta, must vanish-at least in the one-photon approximation. (This was tested at HERMES and no asymmetry at the $10^{-3}$ level was found [9].) As a sizable asymmetry amplitude is seen for the $\pi^{-}$only, which is negative
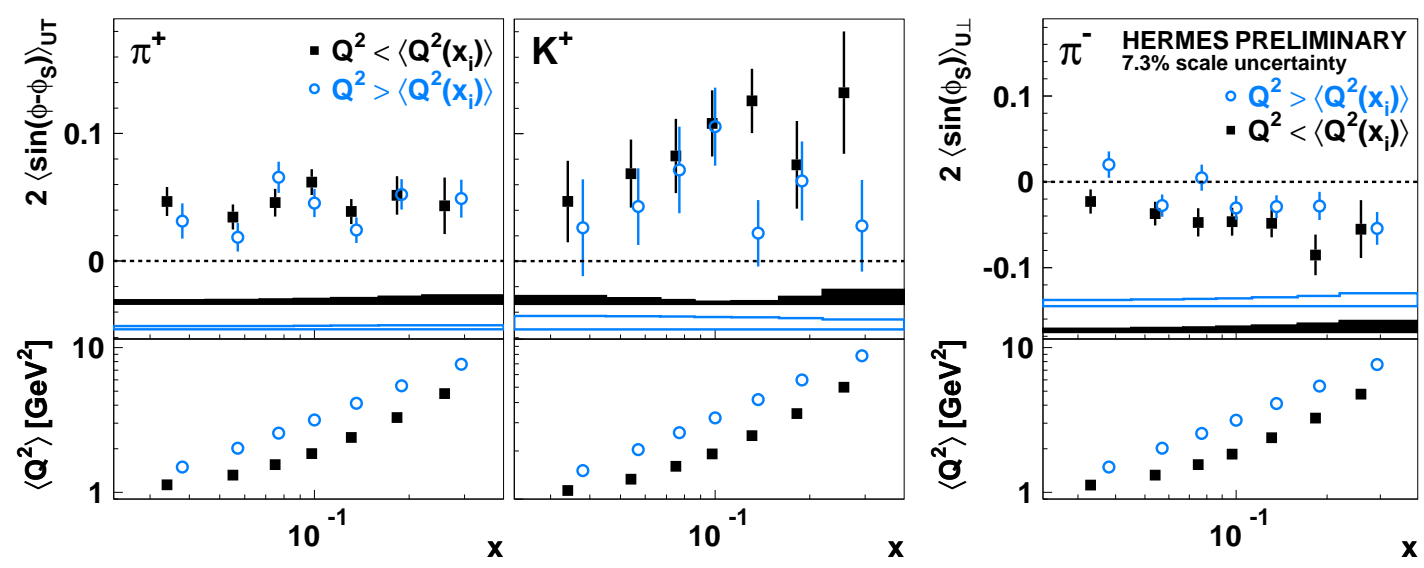

Figure 4: Sivers amplitudes for $\pi^{+}$(left) and $K^{+}$(middle) and the subleading-twist $\sin \phi_{S}$ amplitude (right) as functions of $x$. The $Q^{2}$ range for each bin was divided into the two regions above and below $\left\langle Q^{2}\left(x_{i}\right)\right\rangle$ of that bin. In the bottom the average $Q^{2}$ values are given for the two $Q^{2}$ ranges. 
and does not change sign in the kinematic range examined, the question arises where the missing strength is hidden that is needed to balance out the $\pi^{-}$amplitude to zero. Indeed, a rather large and positive asymmetry was reported for exclusive $\pi^{+}$production at HeRmes [10].

Of the remaining amplitudes on a transversely polarized target, only the Collins asymmetry is significantly non-zero [11]. Therefore, transversely polarized quarks do fragment into hadrons that have a preferred momentum direction transverse to the quark spin as quantified by the Collins fragmentation function. The Collins function also appears in the $\sin \left(3 \phi-\phi_{S}\right)$ modulation, there in conjunction with pretzelosity. In the multipole patterns associated with the various TMDs, pretzelosity is the only one related to a quadrupole deformations. Particular interest in pretzelosity arises also through its modeldependent relation to orbital angular momentum and to the difference between transversity and the helicity distributions. However, the signal observed at HERMES is consistent with zero, either because of cancellations of the various quark flavors, pretzelosity being too small, or because of the additional $P_{h \perp}^{2}$ suppression of the $\sin \left(3 \phi-\phi_{S}\right)$ modulation of the cross section.

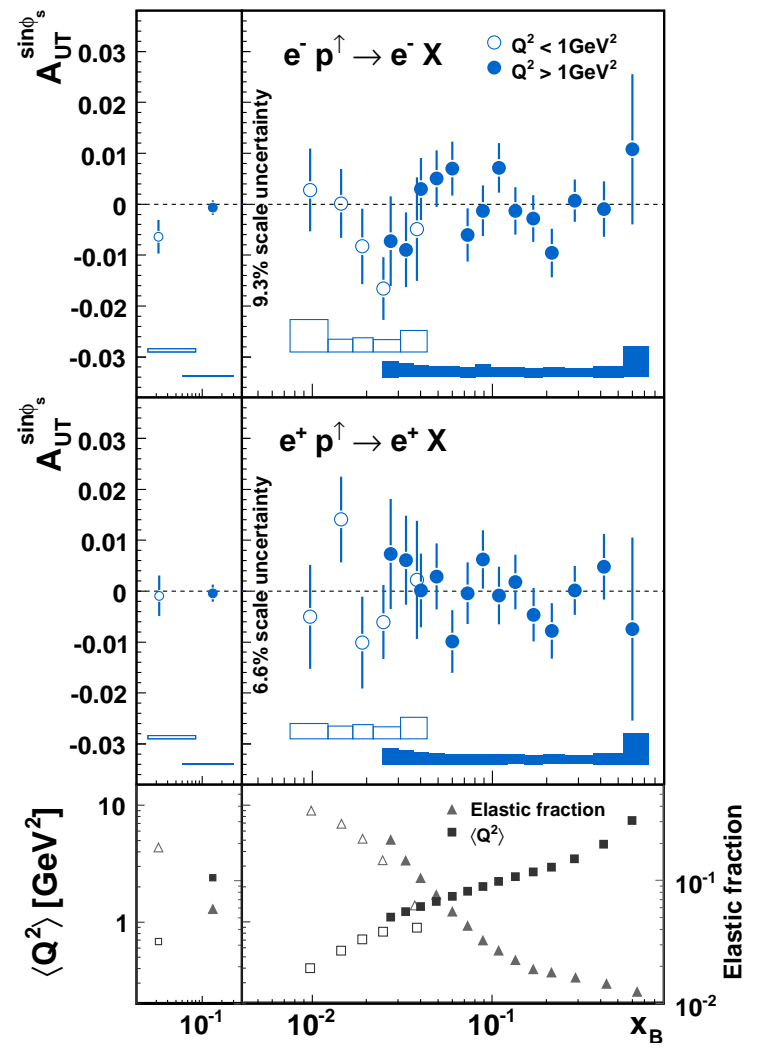

Figure 5: Azimuthal SSA in inclusive DIS off transversely polarized protons.

\section{References}

[1] A. Airapetian, et al., Phys. Rev. Lett. 84 (2000) 4047-4051.

[2] J. C. Collins, Nucl. Phys. B396 (1993) 161-182.

[3] S. J. Brodsky, D. S. Hwang, I. Schmidt, Phys. Lett. B530 (2002) 99-107.

[4] A. Bacchetta, M. Diehl, K. Goeke, A. Metz, P. J. Mulders, M. Schlegel, JHEP 02 (2007) 093.

[5] A. Bacchetta, U. D’ Alesio, M. Diehl, C. A. Miller, Phys. Rev. D70 (2004) 117504.

[6] K. Ackerstaff, et al., Nucl. Instrum. Meth. A417 (1998) 230-265.

[7] A. Airapetian, et al., Phys. Rev. Lett. 103 (2009) 152002.

[8] A. Airapetian, et al., Phys. Lett. B666 (2008) 446-450.

[9] A. Airapetian, et al., Phys. Lett. B682 (2010) 351-354.

[10] A. Airapetian, et al., Phys. Lett. B682 (2010) 345-350.

[11] A. Airapetian, et al., arXiv 1006.4221. 